\title{
Yan-Hou-Qing formula attenuates ammonia-induced acute pharyngitis in rats via inhibition of NF-KB and COX-2
}

\author{
Min Xu' ${ }^{1,2,3 \dagger}$, Tian-Yong Hu${ }^{1 \dagger}$, Dong-Cai Li ${ }^{1}$, Li Ma ${ }^{1}$, Hua Zhang ${ }^{1}$, Jun-Ting Fan ${ }^{4}$, Xiao-Mei Fan ${ }^{5}$, Xian-Hai Zeng ${ }^{1}$, \\ Shu-Qi Qiu', Zhi-Qiang Liu' and Bao-Hui Cheng ${ }^{1 *}$
}

\begin{abstract}
Background: Yan Hou Qing (YHQ) is a Chinese medicinal formula designed to alleviate sore throat symptoms, but underlying mechanism of $\mathrm{YHQ}$ treatment for pharyngitis is poorly defined up to now.

Methods: In this study, the modulation of $\mathrm{YHQ}$ on pharyngitis is investigated in ammonia-induced acute pharyngitis rat models. After treatment with $\mathrm{YHQ}$ or dexamethasone respectively for five consecutive days, all rats were sacrificed for biomolecular and histopathologic study. Protein expressions of MAPKs, NF-KB, COX-2 and 5-LOX in pharyngitis tissue were evaluated by western blot analysis and the levels of TNF-a, IL-6, prostaglandin (PG) $E_{2}$, leukotrienes (LT)-B $B_{4}$ and $L T-D_{4}$ in pharyngeal tissue were measured via ELISA assay. Evans blue (EB) dye exudation test was performed parallelly to assess the integrity of pharyngeal tissue.

Results: Compared with normal control group, EB dye exudation, and inflammatory cytokines in the model group were significantly increased, and the pharynx tissue was obviously infiltrated by inflammatory cells. YHQ treatment improved the inflammatory infiltrate in pharyngeal tissue, and reduced EB dye exudation in AP rat models. The up-regulated TNF- $a$ and IL6 in pharyngeal tissue of AP were significantly reduced by YHQ through inhibition of phosphorylation of p38, Erk and NF-KB. $\mathrm{YHQ}$ treatment also reversed the increased level of $\mathrm{PGE}_{2}$ through down-regulation of COX-2.
\end{abstract}

Conclusions: YHQ formula attenuated the pharyngitis related symptoms via suppression of COX-2 and phosphorylation of p38, Erk and NF-KB (p65).

Keywords: Pharyngitis, Inflammation, Yan Hou Qing, NF-kB; COX-2

\section{Background}

Pharyngitis, characterized by itchy throat and cough, is a common upper respiratory tract disease $[1,2]$. It is estimated that about 600 million cases of symptomatic pharyngitis occur annually worldwide, and pharyngitis prevalence is up to $20 \%$ among children aged $0-14$ years in China [3, 4]. It causes enormous use of health resources

\footnotetext{
* Correspondence: chengbaohui@sina.com

${ }^{\dagger} \mathrm{Min} \mathrm{Xu}$ and Tian-Yong Hu contributed equally to this work.

'Department of Otolaryngology, Longgang E.N.T hospital \& Shenzhen Key

Laboratory of E.N.T, Institute of E.N.T, 3004 Longgang Avenue, Shenzhen

518172, China

Full list of author information is available at the end of the article
}

and the total cost of pharyngitis among children ranges from \$224 to \$539 million per year in the United States [5]. Pharyngitis can be caused by virus, bacteria, smoking, allergic allergen and acid reflux, and antibiotics is recommended for group A streptococcus (GAS)-caused pharyngitis therapy. Clinical management of non-infection pharyngitis remains a matter for debate because of the poor therapeutic effect of antibiotics [6]. It has been well documented that inflammatory responses play a key role in the pathogenesis of non-infection pharyngitis, and repetitive and acute inflammatory insults may render the chronic ear-nose-throat disease and nasopharyngeal

(c) The Author(s). 2020 Open Access This article is licensed under a Creative Commons Attribution 4.0 International License, which permits use, sharing, adaptation, distribution and reproduction in any medium or format, as long as you give appropriate credit to the original author(s) and the source, provide a link to the Creative Commons licence, and indicate if changes were made. The images or other third party material in this article are included in the article's Creative Commons licence, unless indicated otherwise in a credit line to the material. If material is not included in the article's Creative Commons licence and your intended use is not permitted by statutory regulation or exceeds the permitted use, you will need to obtain permission directly from the copyright holder. To view a copy of this licence, visit http://creativecommons.org/licenses/by/4.0/ The Creative Commons Public Domain Dedication waiver (http://creativecommons.org/publicdomain/zero/1.0/) applies to the data made available in this article, unless otherwise stated in a credit line to the data. 
mucosa susceptible to carcinogenesis [7]. So therapeutic interventions against inflammatory may enable protection and treatment non-infection pharyngitis.

Chinese medicine has a long history in treatment of non-infection pharyngitis and attracts much attention of medical worker for its satisfactory therapeutic effect [810]. Yan-Hou-Qing formula (YHQ) is developed for treating respiratory diseases such as laryngopharyngitis by Shenzhen Institute of Ear Nose \& Throat (ENT) and Longgang ENT hospital based on clinical experiences $[11,12]$. Clinical trials for YHQ have been conducted between 2009 and 2014, and YHQ is under the application for approval of China Food and Drug Administration (CFDA) to market a new drug $[13,14]$. This formula is composed of 14 species of medicinal herbs (detailed information see Table 1). Our previous in vitro study revealed that YHQ possess anti-inflammatory activity in lipopolysaccharide (LPS)-stimulated murine macrophages via inhibition of nuclear factor (NF) $-\kappa B$ activity, and in vivo study demonstrated that YHQ attenuate allergic airway inflammation through upregulation of regulatory $\mathrm{T}$ cell (Treg) and suppressing $\mathrm{Th} 2$ responses in Ovalbumin-induced asthmatic mice $[15,16]$. However, underlying mechanisms of YHQ on pharyngitis have not been clarified clearly. Ammonia-induced acute pharyngitis (AP) rat model is a non-infection pharyngitis rat model prevalently used for assessment of the therapeutic effect of complementary and integrative medicine on non-infection pharyngitis. Thus, the aim of present study was designed to determine the underlying mechanisms of YHQ on non-infection pharyngitis based on ammonia-induced AP rat models.

\section{Methods}

\section{Preparation and quality control of YHQ formula}

The botanical origins of these herbal medicines were authenticated by Dr. Yi-Sheng Li, Shenzhen Key Laboratory of ENT, Institute of ENT \& Longgang ENT hospital, Shenzhen, China. All voucher specimens (detailed numbers mentioned in following production procedure) were deposited in the specimen room of
Shenzhen Key Laboratory of ENT, Institute of ENT, Shenzhen, China.

The production procedures of YHQ formula were performed by Guangzhou Kangyuan Pharmaceutical Co., Ltd. (Guangzhou, China) according to the standard operating procedures. Briefly, Lonicera japonica Thunb. (No.2013110201), Scrophularia ningpoensis Hemsl. (No.2013110202), Canarium album (Lour.) DC. (No.2013110203), Sterculia lychnophora Hance (No.2013110204), Terminalia chebula Retz. Hance (No.2013110205) and Siraitia grosvenorii (Swingle) C. Jeffrey ex A. M. Lu \& Zhi Y. Zhang Hance (No.2013110206) were extracted with $7.5 \mathrm{~L}$ boiling water for $2 \mathrm{~h}$ for twice, and then concentrated under reduced pressure at $60^{\circ} \mathrm{C}$. The extract solution of Platycodon grandiflorum (Jacq.) A. DC. (No.2013110207), Belamcanda chinensis (L.) DC. (No.2013110208), Sophora tonkinensis Gagnep (No.2013110209), Tinospora sagittata Gagnep. (No.2013110210) and Physalis alkekengi L. (No.2013110211) were obtained with aqueous ethanol refluxing using $60 \% \mathrm{EtOH}(1 \mathrm{~L} \times 3,1 \mathrm{~h}$ each time). These two extracts were combined and further spray dried with hot air at $150^{\circ} \mathrm{C}$, and the herbal extract ratio was about $23.3 \%$. Spray dried extract mixed with powder of $\mathrm{F}$. Cirrhosa D. Don (No.2013110212) with addition of powder sweetening agent, and was further granulated spraying with ethanol solution of $M$. canadensis $\mathrm{L}$. (No.2013110213) and C. camphora (L.) Presl. (No.2013110214). After tablet coating procedures, the YHQ formula was finally obtained.

Product quality was monitored by high performance liquid chromatography (HPLC). In brief, harpagoside, and cinnamic acid, as the main anti-inflammation components in S. ningpoensis Hemsl. was selected as the chemical markers for quality control [17-19]. Harpagoside and cinnamic acid were prepared in methanol in series of concentrations. 10 tablets of YHQ formula (batch numbers: 140401, 140,403, 140,403, 140,501, 140,502, $140,503,140,601,140,602,140,603)$ were ground into powder, extracted with methanol and fixed to $5 \mathrm{ml}$ in volumetric bottle. All the samples were analyzed using the Waters HPLC 2695 system with photodiode array

Table 1 Formula of $\mathrm{YHQ}$

\begin{tabular}{llll}
\hline Formula YHQ & Amount & Formula YHQ & Amount \\
\hline L. japonica Thunb. & $125 \mathrm{~g}$ & S. tonkinensis Gagnep. & $25 \mathrm{~g}$ \\
S. ningpoensis Hemsl. & $125 \mathrm{~g}$ & B. chinensis (L.) DC. & $25 \mathrm{~g}$ \\
C. album (Lour.) DC. & $125 \mathrm{~g}$ & T. sagittata Gagnep. & $25 \mathrm{~g}$ \\
S. Iychnophora Hance & $125 \mathrm{~g}$ & P. alkekengi L. & $25 \mathrm{~g}$ \\
$\begin{array}{ll}\text { T. chebula Retz. } \\
\text { P. grandiflorum (Jacq.) A. DC. }\end{array}$ & $125 \mathrm{~g}$ & F. cirrhosa D. Don & $25 \mathrm{~g}$ \\
S. grosvenorii (Swingle) C. Jeffrey ex A.M.Lu \& Zhi Y.Zhang & $125 \mathrm{~g}$ & C. camphora (L.) Presl & $2.5 \mathrm{~g}$ \\
\hline
\end{tabular}


detector (Waters Corporation, MA, USA) with Waters Symmetry C18 column $(250 \mathrm{~mm} \times 4.6 \mathrm{~mm}$ i.d., $5 \mu \mathrm{m})$. The mobile phases consisted of acetonitrile (A) and $0.3 \%$ $\mathrm{H}_{3} \mathrm{PO}_{4}$ (B) in a gradient elution: $20-40 \% \mathrm{~A}, 0-15 \mathrm{~min}$; 40-20\% A, 15-15.1 min; 20\% A, 15.1-25 min. The flow rate was $1.0 \mathrm{ml} / \mathrm{min}$ and the column temperature was set at $30^{\circ} \mathrm{C}$. Ten $\mu \mathrm{l}$ of sample was injected and the analytes were monitored with a photodiode array detector (PAD) at the wavelength of $279 \mathrm{~nm}$. The content of harpagoside and cinnamic acid in YHQ formula were $205.5 \mu \mathrm{g} / \mathrm{g}$ (RSD: 0.34\%) and $603.6 \mu \mathrm{g} / \mathrm{g}$ (RSD: 0.29\%), respectively (Fig. 1) [15, 16].

\section{Chemicals and reagents}

Formamide was provided by Sigma-Aldrich (Guangzhou, Guangdong, China). Cell lysis buffer was ordered from Beyotime Biotechnology (Shanghai, China). Antibodies for $\beta$-actin, cyclooxygenase 2 (COX-2), 5-Lipoxygenase (LOX), Erk, Phospho-Erk, JNK, Phospho-JNK, p38, Phospho-p38, IKK $\beta$, Phospho-IKK $\beta$, ІкB $\alpha$, PhosphoI $\mathrm{B} \alpha$ (Ser32/36), NF-кB p65, and phospho-NF-кB p65 were purchased from Cell Signaling Technology, Inc. (Danvers, MA, USA). Relative peroxidase-conjugated secondary antibody, Bicinchoninic acid (BCA) assay, Evans blue (EB) dye and Pierce ${ }^{\mathrm{Tx}}$ ECL Western Blotting Substrate were purchased from Thermo Scientific (Waltham, MA, USA). ELISA for IL-6, TNF- $\alpha, \mathrm{PGE}_{2}, \mathrm{LT}_{-} \mathrm{B}_{4}$ and $\mathrm{LT}-\mathrm{D}_{4}$ were purchased from Cusabio (Wuhan, Hubei, China).

\section{Experimental animals}

Female SD rats (180-220 g) from Guangdong experimental animal center were used for the study. The animals were maintained at $25^{\circ} \mathrm{C}$ in a clean environment under 12: $12 \mathrm{~h}$ light-dark cycle. All rats had free access to food and distilled water. All the experimental protocols were approved by Institutional Animal Care and Use Committee (IACUC) at Shenzhen Institute of ENT (SZENT-2018-012).

\section{Induction of pharyngitis and animal treatment}

Acute pharyngitis (AP) model was established by spraying $15 \%$ ammonia water on the pharynx of rats from day 1 to day 3 (spray 3 thrushes every time, cause the pharynx mucosa to be hyperemia swollen, and form the acute inflammation) and normal control group (NC) was
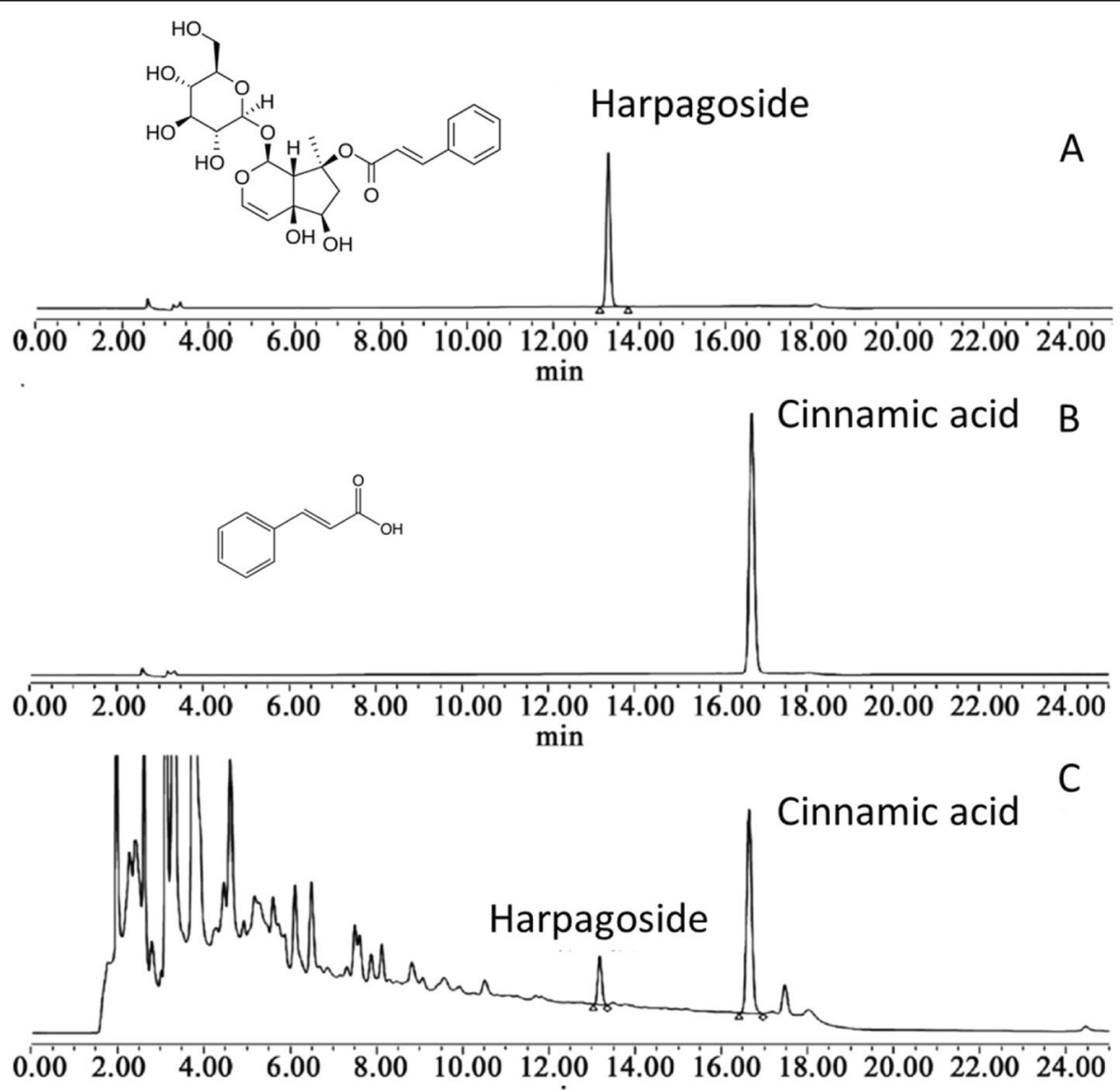

Fig. 1 Representative HPLC-DAD chromatogram (at $279 \mathrm{~nm}$ ) of the chemical standard of harpagoside (a), cinnamic acid (b) and YHQ formula (c) 
sprayed with same volume of saline. The ammonia induced pharyngitis rat models were randomly divided into AP model group (12 rats), YHQ treatment group (12 rats) and dexamethasone treatment group (Dex) (12 rats) on the 4th day. The dosage of YHQ used in ammonia-induced AP treatment is transformed from the clinical used dosage of YHQ (Based on phase I-II clinical trials study, and there is no toxicity observed when rats were oral administrated with YHQ up to dose of $8 \mathrm{~g} / \mathrm{kg}$ ) according to the "Guidance for Industry Estimating the Maximum Safe Starting Dose in Initial Clinical Trials for Therapeutics in Adult Healthy Volunteers" published by U.S. Food and Drug Administration (https://www.fda.gov/media/72309/download). As a steroidal anti-inflammatory drug, long-time oral use of dexamethasone reported with a variety of side effects, so on the premise of ensuring the effective dose of dexamethasone, the dexamethasone dosage should be used as low as possible in ammonia-induced AP. For the treatment group, YHQ formula and dexamethasone were oral administrated $1 \mathrm{~g} / \mathrm{kg}$ and $1 \mathrm{mg} / \mathrm{kg}$ once a day for 5 days, respectively. $\mathrm{NC}$ group and AP group were orally given with same volume of saline (Fig. 2).

\section{Behavioral study and pharyngeal tissue pathological evaluation}

According to behavioral and physical marking criteria (Table 2), scores for diet, activities, mouth scratch, mouth hair, cough and saliva secretion variations for ammonia induced AP rat models after YHQ and dexamethasone treatment were assessed. Scores for mucosal hyperplasia, cell infiltration, vascular dilation \& bleeding and gland hypertrophy changes were also determined based on pathological marking criteria (Table 3) for all rat groups.

Evaluation of pharyngeal mucus integrity via Evans blue dye Parallel to these experiments another set of experiments were run without administration of EB dye. On 8th day after administration of last dose of assigned treatments,
EB dye $(30 \mathrm{mg} / \mathrm{kg}$, i.v.) was administered to all rats in NP, AP, YHQ and Dex group via tail intravenous injection. One hour later, all rats were sacrificed by exsanguination and the head portion was perfused with heparinized saline to expel the intravascular EB dye. After the removal of pharynx tissue, pharynx tissue was cut into pieces, extracted with formamide at $55^{\circ} \mathrm{C}$ for $24 \mathrm{~h}$ and further determined at $620 \mathrm{~nm}$ using SpectraMax Paradigm Multi-Mode Microplate Reader (Molecular Devices, CA, USA).

\section{Determination of inflammatory and pro-inflammatory cytokines in pharynx}

As the typical pro-inflammation cytokines (TNF- $\alpha$ and IL$6)$ and inflammation mediators $\left(\mathrm{PGE}_{2}, \mathrm{LT}-\mathrm{B}_{4}\right.$ and LT- $\left.\mathrm{D}_{4}\right)$ indicating degree of inflammation in ammonia-induced acute pharyngitis rat model, these pro-inflammation cytokines and inflammation mediators in pharyngitis tissue were detected [20]. After homogenization and centrifugation, concentrations of TNF- $\alpha$, IL-6, PGE $2, \mathrm{LT}-\mathrm{B}_{4}$ and LT$\mathrm{D}_{4}$ in supernatant were measured by quantitative sandwich enzyme-linked immunoassay (ELISA) kits (CUSABIO, Wuhan, Hubei, China). All ELISA experiments were strictly performed according to the manufacture's instruction. Absorbance was measured at $450 \mathrm{~nm}$ using SpectraMax Paradigm Multi-Mode Microplate Reader (Molecular Devices, CA, USA).

\section{Western blot analysis of inflammation-related enzymes} As the key proteins involved in triggering inflammation, the protein expressions of arachidonate 5-lipoxygenase (5LOX), cyclooxygenase 2 (COX-2), Erk, phospho-Erk, JNK, phospho-JNK, p38, phospho-p38, IKK $\beta$, phospho-IKK $\beta$, ІкB $\alpha$, phospho-ІкB $\alpha$, NF-кB p65, phospho-NF-кB p65 were probed via western blot [21]. To perform protein extraction, pharyngeal tissues were homogenized and extracted with cell lysis buffer with protease and phosphatase inhibitor cocktail. The protein concentrations

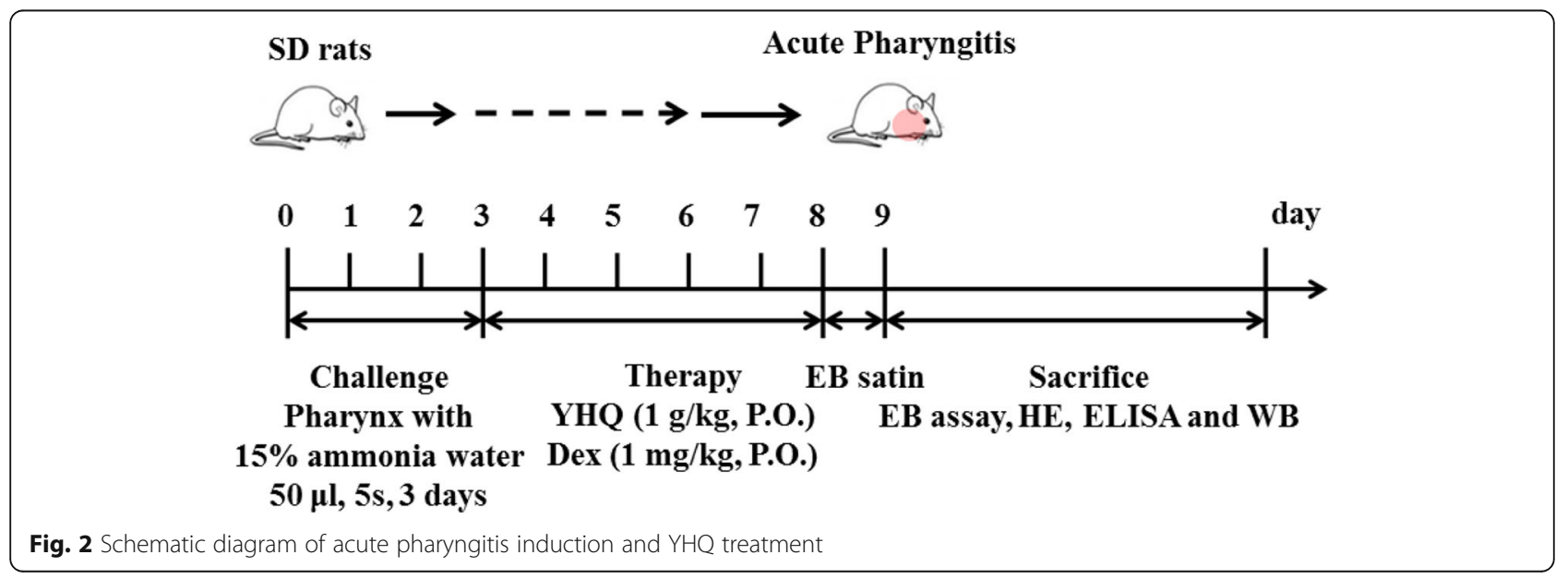


Table 2 Behavioral and physical score criteria for ammonia induced pharyngitis rat models

\begin{tabular}{llllllll}
\hline Symptom rating & Diet reduction & Activities reduction & Mouth scratch & Mouth hair loss & Cough & Saliva secretion & Pharynx swelling \\
\hline 0 & No & No & Normal & No & No & No & Normal \\
1 & Mild & Mild & Few & Few & Mild & little & Mild \\
2 & Obvious & Obvious & Many & Many & Severe & Mass & Severe \\
\hline
\end{tabular}

were determined by BCA assay. Protein samples were then separated by SDS-PAGE containing $8 \%$ of SDS, and electroblotted onto PVDF membranes (GE Healthcare, USA). The membranes were blocked with 5\% BSA solution (in Tris-buffered saline $(150 \mathrm{mM} \mathrm{NaCl}, 20 \mathrm{mM}$ Tris- $\mathrm{HCl}$, $\mathrm{pH}$ 7.6) with $0.1 \%$ Tween 20 ) for $1 \mathrm{~h}$ at room temperature. The membranes were then incubated with primary antibodies against 5-LOX, COX-2, Erk, phospho-Erk, JNK, phospho-JNK, p38, phospho-p38, IKB kinase (IKK) $\beta$, phospho-IKK $\beta$, ІкB $\alpha$, phospho-IкB $\alpha$, NF-кB p65, phospho-NF- $\mathrm{BB}$ p65 and $\beta$-actin at $4{ }^{\circ} \mathrm{C}$ overnight. After washing with Tris buffered saline, peroxidase-conjugated secondary antibodies were added to the membranes and incubated at room temperature for $1 \mathrm{~h}$. The expressions of proteins were detected by ECL reagent, followed by exposure of the membranes to ChemiDoc ${ }^{\mathrm{Tn}} \mathrm{MP}$ Imaging System (Bio-Rad, Hercules, California, USA). The intensity of protein bands was measured by densitometry and quantified using the Image J Software.

\section{HE and immunofluorescence staining}

Pharynx tissue was infused by $4 \%$ paraformaldehyde fixing solution and the specimens were dehydrated and embedded in paraffin. For histological examination, $4 \mu \mathrm{m}$ sections of embedded tissues were cut using a Leica RM2235 rotary microtome (Wetzlar, Germany), placed on glass slides, deparaffinized, and stained sequentially with hematoxylin and eosin (HE). Images of lung tissue sections stained with HE were acquired with a Nikon Eclipse Ci-L/S microscope (Nikon, Tokyo, Japan) equipped with a digital imaging system (Nikon, Tokyo, Japan).

\section{Immunofluorescence and confocal microscopy}

For immunofluorescence staining, pharynx tissue slides were permeabilized with PBS containing 0.3\% Triton X100 and $10 \%$ BSA. Sections of tissues were incubated with NF-kB p65 primary antibodies overnight at $4{ }^{\circ} \mathrm{C}$ and then incubated with FITC labeled secondary antibodies in the dark for $1 \mathrm{~h}$ at $25^{\circ} \mathrm{C}$. Cell nuclei were stained using DAPI for $10 \mathrm{~min}$, and fluorescent images were captured with LEICA TCS SP5 II confocal microscope (Leica Microsystems Inc. Buffalo Grove, IL, USA).

\section{Statistical analysis}

Behavioral and physical scores and pathological scores were exhibited as median with interquartile rage, and other results were expressed as mean \pm SEM. Each experiment was repeated thrice in triplicate. Multiple group comparisons were performed using one-way ANOVA followed by Tukey test using GraphPad Prism 5.01 (La Jolla, CA, U.S.A.). The differences were considered as statistically significant when $p<0.05$.

\section{Results}

\section{Pathological improvement of YHQ on pharynx in AP rat models}

After modeling, the frequencies of mouth scratch and cough increased, and food intake behaviors decreased (details see Fig. 3). Pharynx showed redness and swelling and accumulated mucous secretions, and superficial ulcer was formed. Some of the AP rat models lost their mouth hair gradually. The normal control group had normal autonomic activity, and there was no ulceration in pharyngeal mucosa.

According to the pharyngitis pathological score criteria, the rat pharynx tissues in each group were assessed (Tables 2 and 3). As shown in Fig. 3, the pathologies of the pharyngeal mucosa and tissue were changed significantly $(p<0.0001)$ in the model group. In model group, the mucous membranes of the pharyngeal tissue of rat form ulcers, and hyperemia and infiltration of inflammatory cells can be seen under the mucous membranes of ulcers. Pharyngeal inflammatory reaction of rats in the $\mathrm{NC}$ group show no the appearance of inflammation, and mucosal and submucosal mucosal glands of the pharyngeal tissue of rats were normal. The inflammatory

Table 3 Pathological score criteria for ammonia induced pharyngitis rat models

\begin{tabular}{lllll}
\hline Score criteria & Mucosal hyperplasia & Cell infiltration & Vascular dilation \& Bleeding & Gland hypertrophy \\
\hline 0 & No & No & No & No \\
1 & Mild & Mild & Mild & Mild \\
2 & Moderate & Moderate & Moderate & Moderate \\
3 & Severe & Severe & Severe & Severe \\
\hline
\end{tabular}




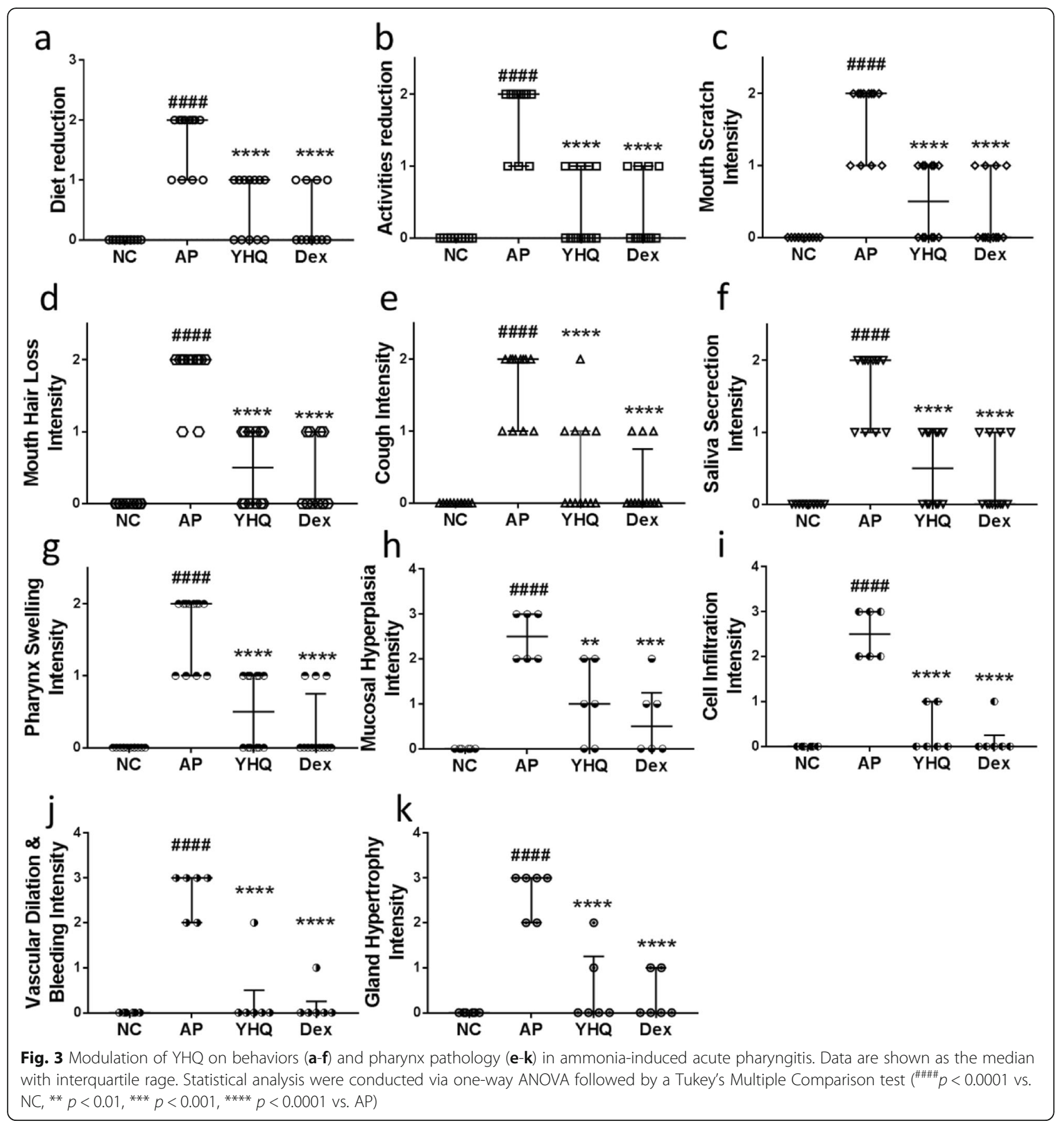

symptoms of pharyngeal tissue in each YHQ group and Dex group improved in different degrees.

YHQ reduces EB dye lesion in pharyngitis tissue via maintaining mucosal integrity

On 8th day, $1 \mathrm{~h}$ after administration of EB dye (30 $\mathrm{mg} / \mathrm{kg}$, i.v.) the pharyngeal tissue was separated and the quantity of EB dye present in the pharyngeal tissue was quantified by using standard curve for EB dye (Fig. 4). Dexamethasone and YHQ treated animals showed macroscopic reduced blue ting, as an indication of their protective effect against ammoniainduced damage (Fig. 4a). AP group showed severe extravasation of EB dye due to inflammation of pharynx, however NC animals applied with saline showed almost no extravasation of EB dye (Fig. 4b). The severe extravasation $E B$ dye levels of pharyngeal tissue were attenuated significantly after 5 days' treatment with YHQ and dexamethasone $(p<0.01$ and $p<$ 0.001 , respectively). 


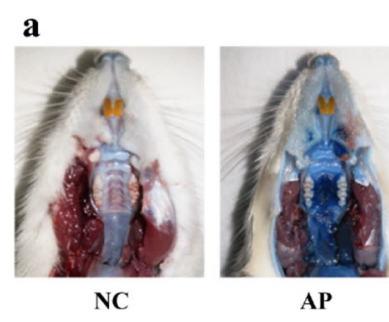

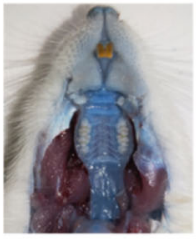

YHQ b
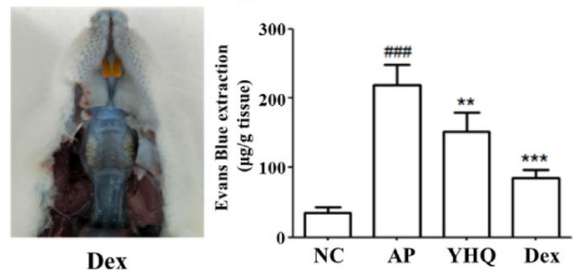

Fig. 4 Effect of $\mathrm{YHQ}$ on ammonia-induced morphological damage of rat pharynx. a Evans Blue (EB) dye lesion due to morphological damage of rat pharynx; $\mathbf{b}$ Variations of EB dye in pharyngeal tissue. Data are shown as the mean \pm SEM. Statistical analysis were conducted via one-way ANOVA followed by a Tukey's Multiple Comparison test $\left({ }^{\# \# \# \#} p<0.0001\right.$ vs. NC; ${ }^{* *} p<0.01,{ }^{* * *} p<0.001$ vs. AP)

YHQ attenuates the cell infiltration in ammonia induced pharyngitis rat model

Histopathological changes on pharynx tissue were assessed by $H E$ staining. H\&E results showed of submucosal gland hypertrophy and thickened mucosa in pharynx of AP rats (Fig. 5), and NC rats showed normal cytoarchitecture of the pharynx. Dexamethasone $(1 \mathrm{mg} /$ kg, p.o.) treated animals showed mild hypertrophy of mucus glands and thin mucosa, especially in improving hypertrophy of mucus gland. Compared to Dex group, YHQ (1 g/kg, p.o.) treated animals showed milder hypertrophy of mucus glands and thinner mucosa which was similar to the NC group (Fig. 5). Noteworthy, YHQ (1 g/ $\mathrm{kg}$, p.o.) were found to be more potent than reference standard dexamethasone $(1 \mathrm{mg} / \mathrm{kg}$, p.o.) in reducing submucosal gland hypertrophy and mucosa thickness in AP rats.

Down-regulation of YHQ on proinflammatory cytokines in pharyngitis rat models

As pro-inflammatory cytokines, interleukin (IL)-6 and tumor necrosis factor (TNF)- $\alpha$ are involved in acute inflammation and make up the acute phase reaction [22]. Compared with the normal control group, the levels of TNF- $\alpha(p<0.001$ and $p<0.05$, respectively, Fig. 6) and IL-6 ( $p<0.001$ and $p<0.05$, respectively, Fig. $6)$ in model group increased significantly, and this upregulations of TNF- $\alpha(p<0.001, p<0.01$, Fig. 6) and IL$6(p<0.001, p<0.01$, Fig. 6$)$ were alleviated after treatment with YHQ or dexamethasone. The inhibition of YHQ on TNF- $\alpha$ and IL- 6 implied that alleviation of YHQ on pharyngitis may depend on down-regulation of inflammatory mediators.

\section{YHQ treatment reduces $L T-B_{4}$ and $L T-D_{4}$ in pharyngitis rat model}

For the down-regulation of YHQ on pro-inflammatory cytokines, the inhibitory effects of YHQ were further evaluated on inflammatory mediators $\left(\mathrm{LT}-\mathrm{B}_{4}\right.$ and LT$\mathrm{D}_{4}$ ) and related synthesized protein 5-LOX. As a fundamental regulator in inflammation process, aiming at inhibition of 5-LOX has been deliberated as an effective approach to prevent inflammatory disorders. Effect of YHQ was evaluated on 5-LOX mediated production of

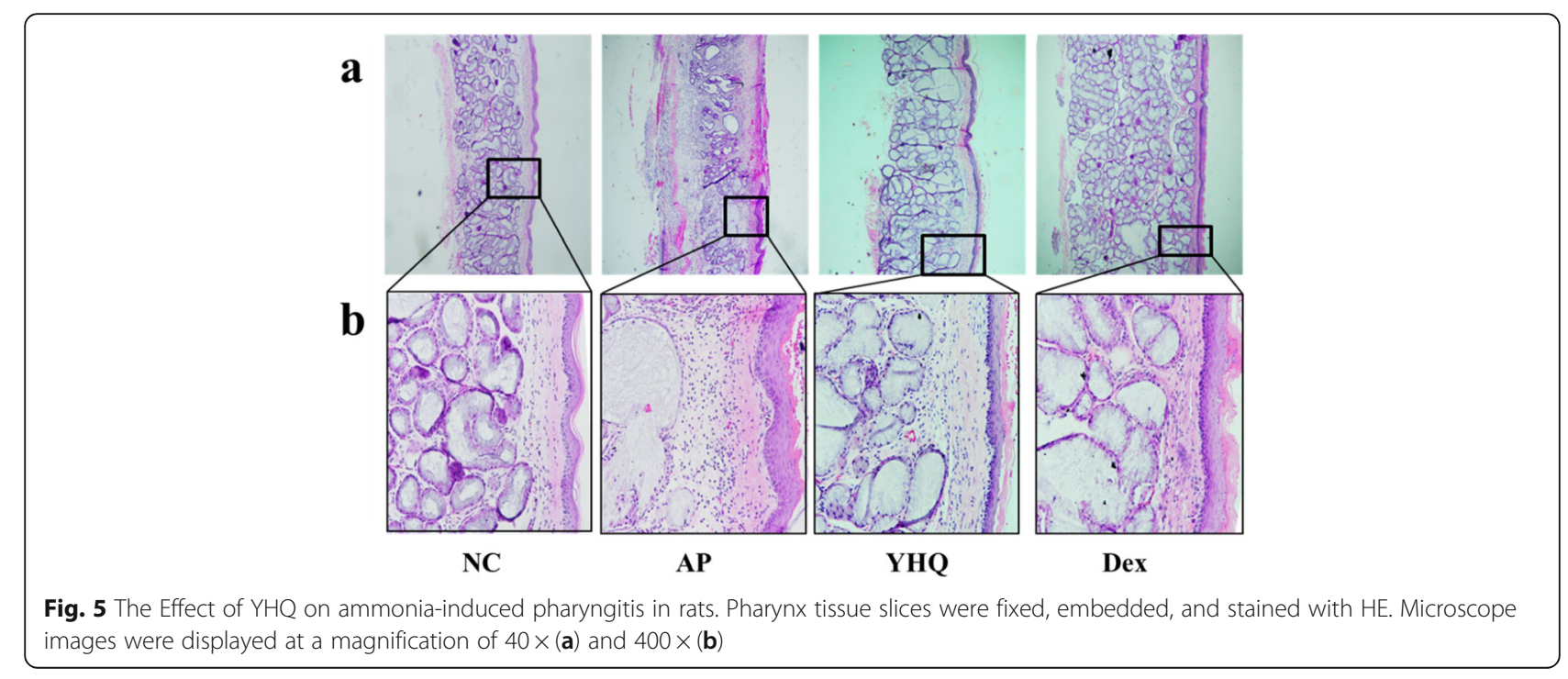



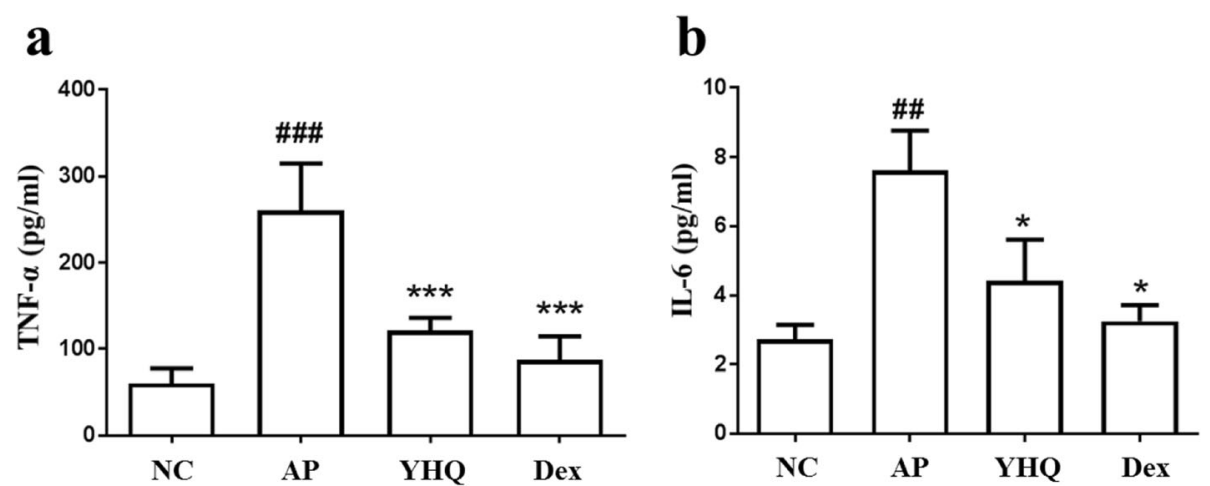

Fig. 6 The effects of YHQ on TNF-a and IL-6 levels in pharynx tissue of rats. (a) Levels of TNF-a; (b) Levels of IL-6. Data are shown as the mean \pm SEM. Statistical analysis were conducted via one-way ANOVA followed by a Tukey's Multiple Comparison test ${ }^{\# \#} p<0.01,{ }^{\# \# \#} p<0.001$ vs. NC; ${ }^{*} p<$ $0.05,{ }^{* *} p<0.001$ vs. AP)

LT- $\mathrm{B}_{4}$ and LT- $\mathrm{D}_{4}$ in acute pharyngitis rat model. ELISA analysis (Fig. 7a and b) showed that ammonia sprays upregulated the release of $\mathrm{LT}-\mathrm{B}_{4}$ and $\mathrm{LT}-\mathrm{D}_{4}$ in pharynx which was significantly attenuated after treatment with YHQ at a dose of $1 \mathrm{~g} / \mathrm{kg}(p<0.001$ and $p<0.01$, respectively). As the synthesis protein for LT- $\mathrm{B}_{4}$ and $\mathrm{LT}-\mathrm{D}_{4}$, the expressions of 5-LOX in pharynx tissue were investigated by western blot, and 5-LOX protein was considerably upregulated in the ammonia induced pharyngitis compared to normal control group. As illustrated in Fig. $6 \mathrm{c}$, the expression of 5-LOX stimulated by ammonia spray, were significantly suppressed after treatment with dexamethasone ( $p<0.01$, Fig. 7c). Compared to AP group, after the treatment with YHQ at $1 \mathrm{~g} / \mathrm{ml}$, and the 5 -LOX protein expressions were decreased by $4.5 \%$, and YHQ show no significant inhibition on 5-LOX $(p>0.05$, Fig. 7c).

\section{Suppression of $\mathrm{YHQ}$ on $\mathrm{PGE}_{2}$ via reduction of $\mathrm{COX}-2$ expression}

$\mathrm{PGE}_{2}$, an arachidonic acid derivative generated by cyclooxgenase-2 (COX-2), is another principal mediator of acute inflammation. ELISA was performed to investigate whether $\mathrm{YHQ}$ suppress $\mathrm{PGE}_{2}$ production in ammonia-

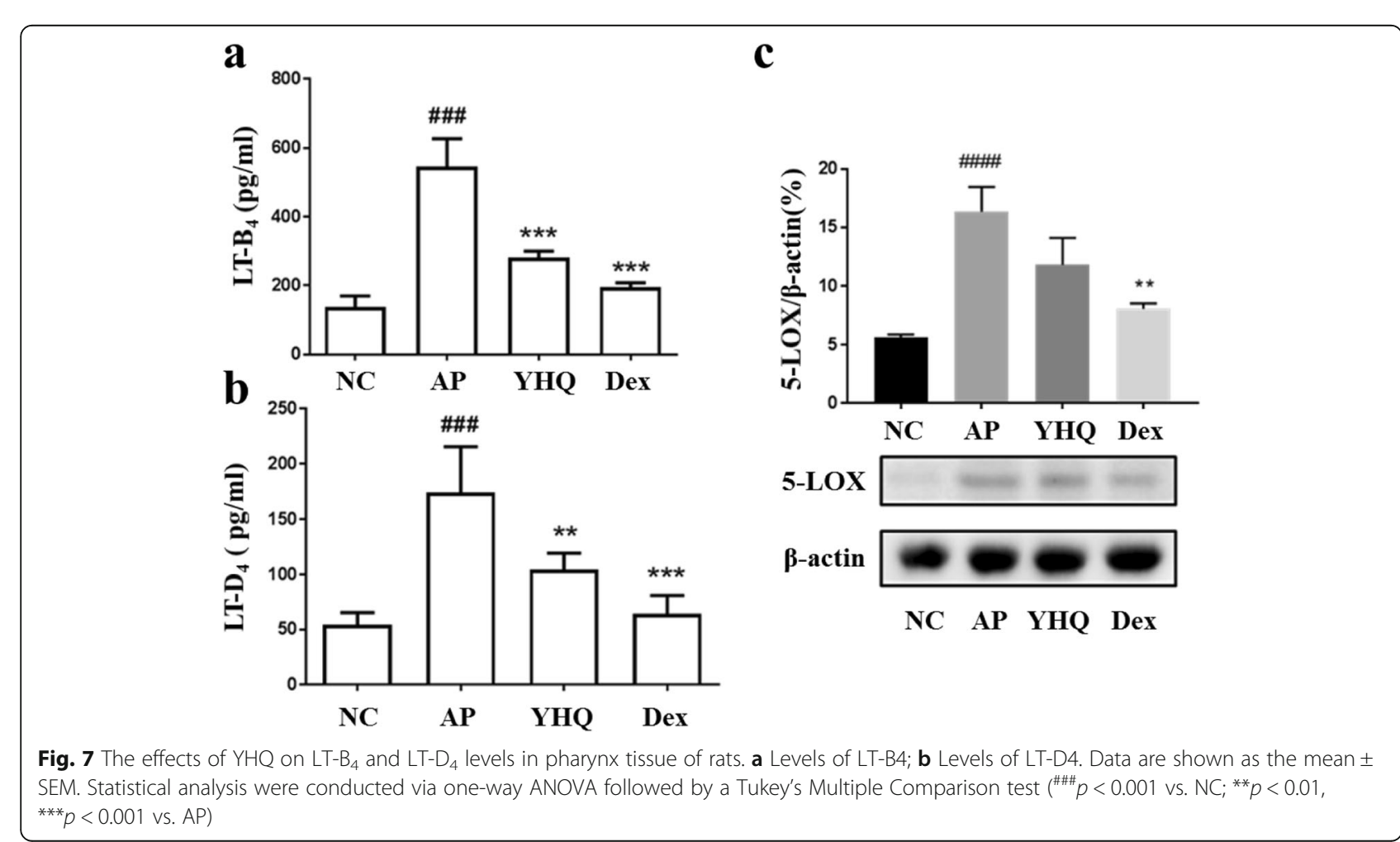



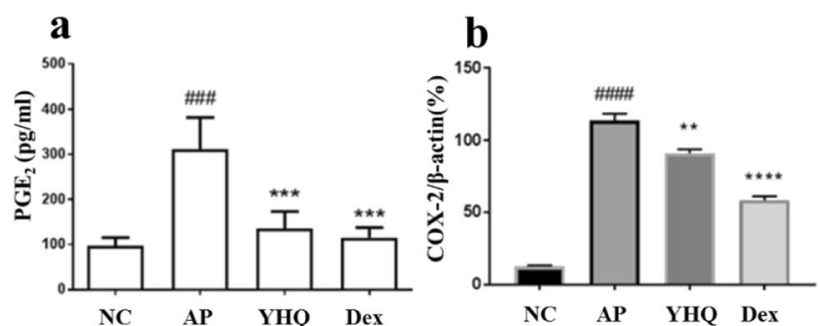

c

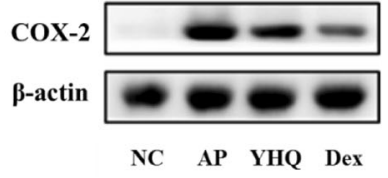

Fig. 8 The effects of YHQ on PGE2 levels and COX-2 expression in pharynx tissue of rats. Data are shown as the mean \pm SEM. Statistical analysis were conducted via one-way ANOVA followed by a Tukey's Multiple Comparison test (\#\#\# $p<0.001$ vs NC; ${ }^{* * *} p<0.001$ vs. AP)

induced pharyngitis. YHQ was found to decrease the level of $\mathrm{PGE}_{2}$ significantly at dose of $1 \mathrm{~g} / \mathrm{kg}$ (Fig. 8a). As shown in Fig. 8b and c, YHQ significantly reduced COX-2 expression $(p<0.01)$ at dose of $1 \mathrm{~g} / \mathrm{kg}$. Compared to pharyngitis group, after the treatment with YHQ at $1 \mathrm{~g} / \mathrm{kg}$, and the COX-2 protein expression was decreased by $22.7 \%$. This result suggests that suppression of YHQ on $\mathrm{PGE}_{2}$ production is related to the regulation of the expression of its synthesis enzyme COX-2 in ammonia-induced pharyngitis.

Nevertheless, these outcomes recommend that the inhibitory effects of YHQ on 5-LOX/LT- $\mathrm{B}_{4} / \mathrm{LT}-\mathrm{D}_{4}$ and $\mathrm{COX}-2 / \mathrm{PGE}_{2}$ are consistent with its inhibitory effects on pro-inflammatory cytokines (IL-6 and TNF- $\alpha$ ) in ammonia-induced pharyngitis.

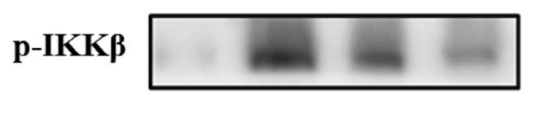

IKK $\beta$

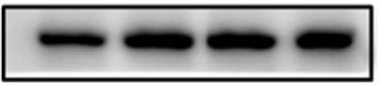

$\mathbf{a}$
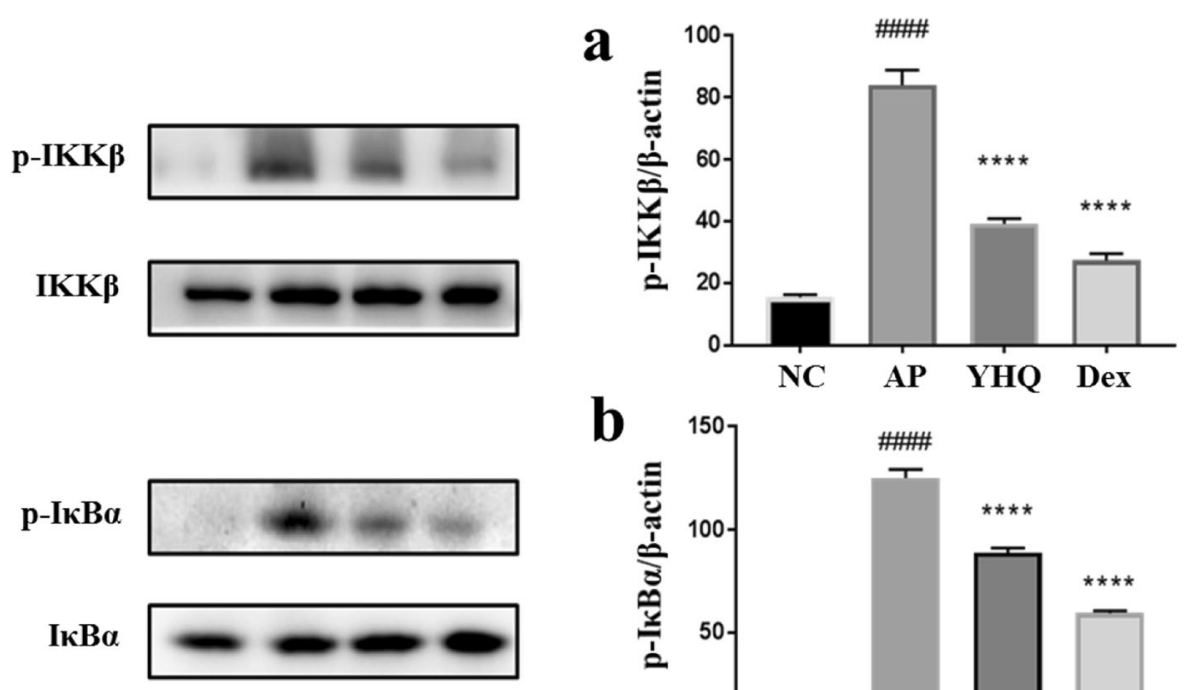

b
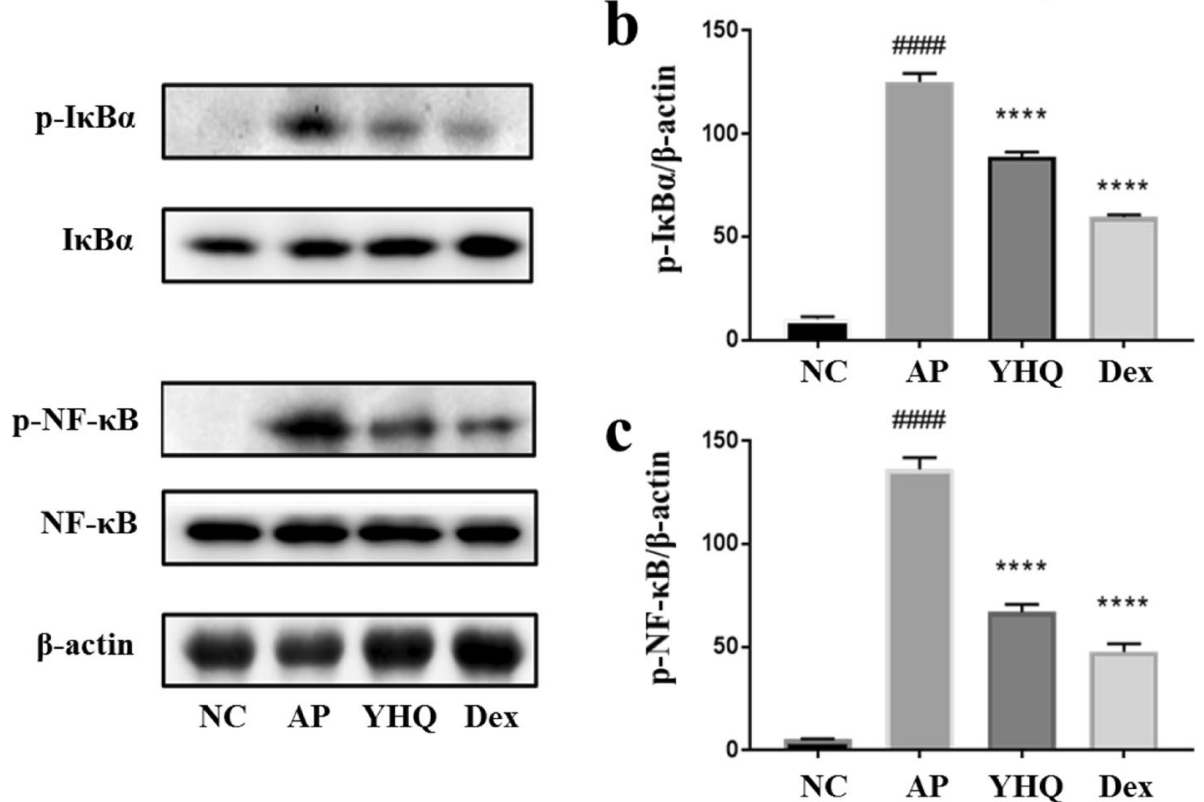

Fig. 9 Effects of $\mathrm{YHQ}$ on phosphorylated IKK $(\mathrm{p}-\mathrm{IKK} \beta)$, $\mathrm{p}-\mathrm{IKB}$ a and $\mathrm{p}-\mathrm{NF}-\mathrm{KB}$ expression in pharynx tissue of rats. Data are shown as the mean \pm SEM. Statistical analysis were conducted via one-way ANOVA followed by a Tukey's Multiple Comparison test (\#\#\#\# $p<0.0001$ vs. NC; ${ }^{* * * *} p<0.0001$ vs. AP) 
Impact of YHQ on MAPKs and NF-KB signaling pathway mediated inflammation in ammonia-induced pharyngitis Tissue damages through the intracellular signaling cascade activates a trimeric IKK complex and then phosphorylates $\mathrm{I} \kappa \mathrm{B} \alpha$ resulting in nuclear translocation of the phosphor-NF- $\mathrm{B}$ for further the transcription of the genes encoding pro-inflammatory cytokines and chemokines $[23,24]$. The IKK enzyme complex is a part of the upstream NF- $\mathrm{kB}$ signal transduction cascade, so YHQ was evaluated for its effect on $\mathrm{pIKK} \beta / N F-\kappa B$ signaling

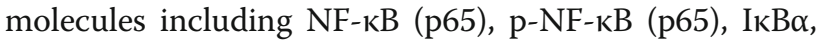
$\mathrm{p}-\mathrm{I} \kappa \mathrm{B} \alpha, \mathrm{IKK} \beta$ and $\mathrm{p}-\mathrm{IKK} \beta$ in pharyngeal mucous tissue of ammonia induced pharyngitis. As compared to the NC group, stimulation with ammonia enhanced the phosphorylation of $\mathrm{p} 65, \mathrm{p}-\mathrm{I} \kappa \mathrm{B} \alpha$ and $\operatorname{IKK} \beta$ (Fig. 9).
Treatment with YHQ showed significant inhibition on $\mathrm{p}-\mathrm{NF}-\mathrm{\kappa B}$ (p65) in respect to ammonia-induced pharyngitis $(p<0.0001$ for $1 \mathrm{~g} / \mathrm{kg}$, Fig. 9c). The modulation of YHQ on NF- $\mathrm{B}$ signal transduction in ammonia-induced pharyngitis rat model depended on prevention of YHQ on $\mathrm{p}-\mathrm{I} \kappa \mathrm{B} \alpha$ and $\mathrm{p}-\mathrm{IKK} \beta$. Suppression of YHQ on $\mathrm{p}-\mathrm{NF}-\mathrm{kB}$ (p65) was via inhibiting $\mathrm{p}$ $\mathrm{IKK} \beta$ and $\mathrm{p}-\mathrm{I} \kappa \mathrm{B} \alpha$ upregulated by ammonia stimulation ( $p<0.001$ and $p<0.001$, respectively) without interfering the level of $\mathrm{I} \kappa \mathrm{B} \alpha$, IKK $\beta$ and NF- $\mathrm{KB}$ p 65 in rat models (Fig. 9a and b). We also confirmed this result via confocal microscope and immunofluorescence results indicated that fluorescence intensity in cell nuclei of pharynx induced by ammonia is apparently suppressed by YHQ suggesting that YHQ down-

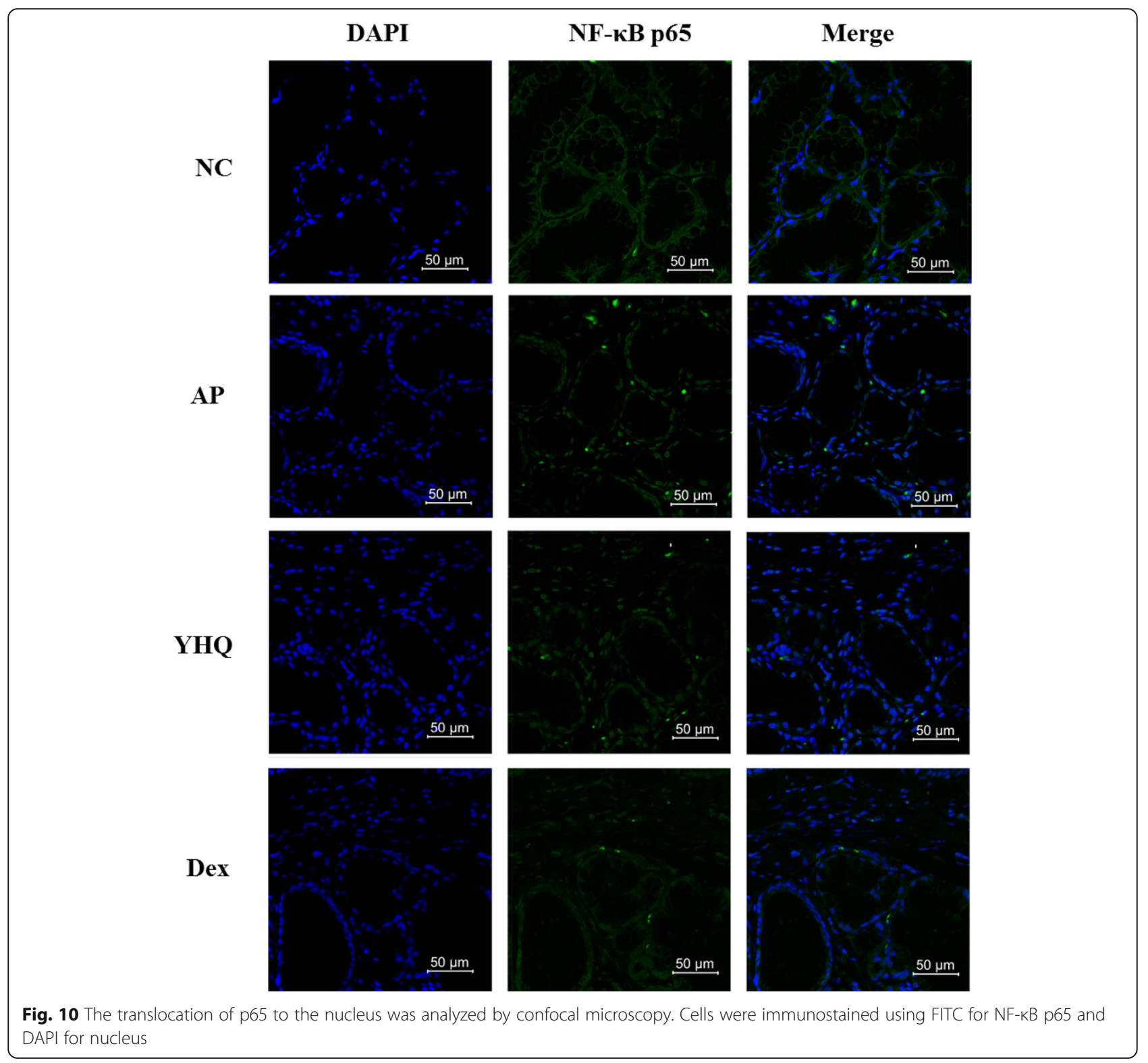


regulated the expression of NF- $\mathrm{kB}$-regulated genes at the transcriptional level (Fig. 10).

The MAPK signaling cascades are well-thought-out to be involved in this molecular mechanism $[25,26]$. During inflammatory processes, phosphorylation of MAPKs (JNK, ERK, and p38) implement critical role by triggering the regulation of NF- $\mathrm{kB}$ signaling pathway. As shown in Fig. 11, ammonia stimulation enhanced phosphorylation of Erk, JNK and p38 protein kinases, and YHQ treatment at dose of $1 \mathrm{~g} / \mathrm{kg}$ inhibits the ammonia induced phosphorylation levels of p38 and Erk in acute pharyngitis rat models, while there is no significant change in protein expression of JNK and p-JNK (Fig. 11c).

\section{Discussion}

This study assessed the effects YHQ formula on pharyngitis via ammonia induced AP rat models, and our data showed that YHQ relieve pharyngitis related symptoms, such diet reduction, activities reduction, mouth scratch, mouth hair loss, cough, saliva secretion, pharynx swelling (Fig. 3a - f), and YHQ also attenuate mucosal hyperplasia, cell infiltration, vascular dilation and gland hypertrophy (Fig. 3g - k). These results indicated that YHQ could effectively improve ammonia induced acute pharyngitis in rat model. YHQ lowered histopathology scores in pharynx, showing that YHQ could reduce the occurrence of hypertrophy gland and mucosa thickness, and relieve ammonia induced pharynx related symptoms.

Our data show that reverse the up-regulated proinflammatory cytokines TNF- $\alpha$, IL- 6 induced by tissue injury due to ammonia sprays. Treatment with YHQ could decrease the expressions of COX-2 and 5-LOX to further reduce the production of inflammatory cytokines $\mathrm{PGE}_{2}, \mathrm{LTB}_{4}$ and $\mathrm{LTD}_{4}$. As the main regulator of proinflammation and inflammation cytokines production (e.g. TNF- $\alpha, \mathrm{IL}-6, \mathrm{PGE}_{2}, \mathrm{LTB}_{4}$ and $\mathrm{LTD}_{4}$ ), the activation of NF- $k B$ is a hallmark of host cells responding to ammonia induced tissue injury. Current study demonstrated that YHQ could attenuate inflammation caused by tissue injury in pharyngitis rat model via inhibiting p38/Erk/ $\mathrm{NF}-\mathrm{kB} / \mathrm{COX}-2$ signaling pathway.

The main mechanisms for current available single compound, herbal extracts and herbal formula for AP treatment are mainly involved in the anti-inflammation [27-29] . YHQ formula was designed by otorhinolaryngology physicians based on traditional Chinese medicine
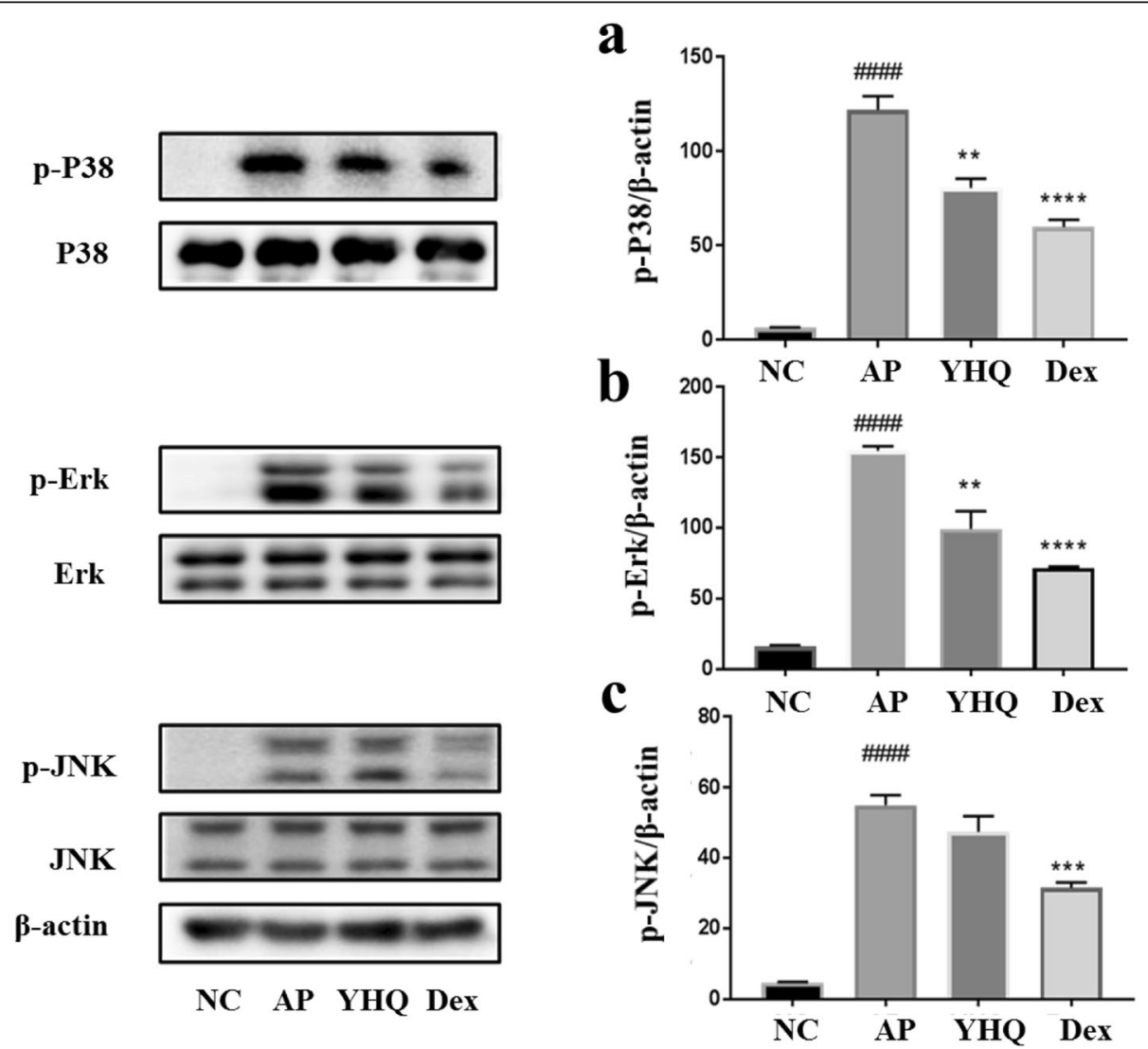

Fig. 11 Effects of $Y H Q$ on phosphorylated p38 (p-p38), p-Erk and p-JNK expression in pharynx tissue of rats. Data are shown as the mean \pm SEM Statistical analysis were conducted via one-way ANOVA followed by a Tukey's Multiple Comparison test (\#\#\#\# $p<0.0001$ vs. NC; ${ }^{* *} p<0.01,{ }^{* * *} p<$ $0.001,{ }^{* * *} p<0.0001$ vs. AP) 
theory and clinic experiences, and YHQ was used as hospital preparation in form of buccal tablet at clinics. It can not only play a direct anti-inflammatory effects, but also benefit the damaged pharyngeal tissue repair. Based on phase I-II clinical trials study, and there is no toxicity observed when rats were oral administrated with YHQ up to dose of $8 \mathrm{~g} / \mathrm{kg}$. It contains 14 kinds of herbal medicines, and individual herbs involved in YHQ have been documented to have various biological effects [15]. Lonicera japonica Thunb. (Jin-Yin-Hua) and Scrophularia ningpoensis Hemsl. (Xuan-Shen) are major components in this formula possessing anti-inflammation [30-32]. Sterculia lychnophora Hance (Pang-Da-Hai), Terminalia chebula Retz. (He-Zi), Canarium album (Lour.) DC. (Qing-Guo), and Belamcanda chinensis (L.) DC. (SheGan) can help the above major components in treatment of pharyngitis via wound healing, antibacteria, and antioxidation to improve pharyngitis symptoms [29, 33, 34]. Siraitia grosvenorii (Swingle) C. Jeffrey ex A.M.Lu \& Zhi Y. Zhang (Luo-Han-Guo), Physalis alkekengi L. (JinDeng-Long), Tinospora sagittata Gagnep. (Jin-GuoGan), Fritillaria cirrhosa D. Don (Chuan-Bei-Mu), Sophora tonkinensis Gagnep. (Shan-Dou-Gen) and Platycodon grandiflorum (Jacq.) A. DC. (Jie-Geng) function as assistant roles (anti-tussive, anti-inflammation, antimicrobial, analgesic and tracheobronchial relaxant) in tissue injury recover during the treatment of pharyngitis [35-40]. Mentha canadensis L. (Bo-He) acts as penetration enhancer promote other herbal medicine absorption and also improve the throat discomfort during pharyngitis [41]. Although YHQ showed no such strong anti-inflammatory effect as reference drug dexamethasone, combination of these 14 herbal medicines exhibited better reduced gland hypertrophy and thinner mucosa in HE staining than dexamethasone used alone and showed similar improved pharyngitis symptoms as dexamethasone.

\section{Conclusions}

This study demonstrated that YHQ possess the remarkable anti-inflammatory effects, protect the mucosal tissue integrity, and improve the pharyngitis symptoms. The therapeutic effects of YHQ on AP are associated with the reduction of pro-inflammatory cytokines (TNF- $\alpha$ and IL-6) and inflammatory mediators $\left(\mathrm{PGE}_{2}, \mathrm{LTB}_{4}\right.$ and $\left.\mathrm{LTD}_{4}\right)$ caused by injuries induced by ammonia in rat model via inhibiting phosphorylation of p38, Erk, and NF- $\mathrm{kB}$ (p65) and lowering the protein expressions of COX-2 and 5-LOX. Our findings support the further evaluation of YHQ as a potential drug for the treatment of non-infection AP. YHQ may have significant clinical potential as a therapeutic agent for the treatment of upper airway inflammatory conditions such as pharyngitis.

\section{Supplementary information}

Supplementary information accompanies this paper at https://doi.org/10. 1186/s12906-020-03077-1.

\section{Additional file 1.}

\section{Abbreviations}

$\mathrm{PGE}_{2}$ : prostaglandin (PG) $\mathrm{E}_{2}$; LT: leukotrienes; EB: Evans blue; YHQ: Yan-HouQing formula; CFDA: China Food and Drug Administration;

LPS: lipopolysaccharide; Treg: Regulatory T cell; NF-kB: Nuclear factor-kB;

HPLC: high performance liquid chromatography; COX-2: cyclooxygenase 2; 5LOX: 5-Lipoxygenase; AP: Acute pharyngitis; NC: control group;

Dex: dexamethasone treatment group; i.v.: intravenous; ELISA: enzyme-linked immunoassay; H\&E: Hematoxylin and eosin stain; IL: interleukin; IKK: IKB kinase

\section{Acknowledgments}

Not applicable.

\section{Authors' contributions}

XM, HTY, ML and ZH acquired, analyzed the data; ZXH and LDC interpreted and performed statistical analysis; FXM and FJT took charge of quality control of YHQ; LZQ, QSQ, and CBH conceived and designed the study. All authors drafted the manuscript. The author(s) read and approved the final manuscript.

\section{Funding}

This work was supported by grants Natural Science Foundation of China (No. 81973915 and 81773978), Guangdong Basic and Applied Basic Research Foundation (No. 2019A1515010391 and 2020A1515010592) and Shenzhen Innovation of Science and Technology Commission (No. SZXK039, LGKCZSYS2019000046, JCYJ20170412103841386, and 2017040320570626). The funding bodies were not involved in the concept, design, analysis or writing the manuscript.

\section{Availability of data and materials}

The data used or analyzed during the current study are available from the corresponding author on reasonable request.

\section{Ethics approval and consent to participate}

Rats were ordered from Guangdong experimental animal center (Production License Number: SCXK (YUE)2013-0002, Use License Number: SYXK (YUE)20140140). Animal experiments were approved and performed in accordance with the guidelines of the Ethics Committee of the Shenzhen institute of ENT and Longgang ENT Hospital, Shenzhen, China (No.2020-0101).

The SD rats used in this study were ordered from Guangdong experimental animal center (license number for experimental animal production:), and this animal center possess animal production certificate in China.

\section{Consent for publication}

All authors have read and approved the final manuscript.

\section{Competing interests}

The authors declare no conflicts of interest.

\section{Author details}

${ }^{1}$ Department of Otolaryngology, Longgang E.N.T hospital \& Shenzhen Key Laboratory of E.N.T, Institute of E.N.T, 3004 Longgang Avenue, Shenzhen 518172, China. 'Zunyi Medical University, Zunyi 563000, Guizhou, China. ${ }^{3}$ Department of Otolaryngology, The third hospital of Mianyang, Mianyang 621000, China. ${ }^{4}$ Department of Pharmaceutical Analysis, School of Pharmacy, Nanjing Medical University, Nanjing 211166, China. ${ }^{5}$ Baoan Maternal and Child Health Hospital, Jinan University, Shenzhen 518102, China.

Received: 28 July 2020 Accepted: 8 September 2020

Published online: 14 September 2020

\section{References}

1. Weber R. Pharyngitis. Prim Care Clin Off Pract. 2014;41(1):91-8.

2. Ruppert SD, Fay VP. Pharyngitis: soothing the sore throat. Nurs Pract. 2015; 40(7):18-25. 
3. Leone C, Caruso AA, Allocca V, Barra E, Leone R. Pilot study on the effects of high molecular weight sodium hyaluronate in the treatment of chronic pharyngitis. Int J Immunopathol Pharmacol. 2015;28(4):532-8.

4. Wu S, Peng X, Yang Z, Ma C, Zhang D, Wang Q, Yang P: Estimated burden of group a streptococcal pharyngitis among children in Beijing, China. In: BMC Infect Dis. vol. 16; 2016: 452.

5. Pfoh E, Wessels MR, Goldmann D, Lee GM. Burden and economic cost of group a streptococcal pharyngitis. Pediatrics. 2008;121(2):229-34.

6. Renner B, Mueller CA, Shephard A. Environmental and non-infectious factors in the aetiology of pharyngitis (sore throat). Inflamm Res. 2012;61(10):104152.

7. Xiao X, Zhang Z, Chang ET, Liu Z, Liu Q, Cai Y, Chen G, Huang QH, Xie SH, Cao SM, et al. Medical history, medication use, and risk of nasopharyngeal carcinoma. Am J Epidemiol. 2018;187(10):2117-25.

8. H-I J, She B, Liu W, Mao B, Zhang J-Y. Efficacy and safety of Qi-Wei-Qing-Yan aerosol in treatment of acute pharyngitis (lung-stomach excess-heat syndrome): study protocol for a randomized controlled trial. Trials. 2016; 17(99):1-10.

9. Lu C, Song Y, Zhang J, Du Y, Wang T, Xue Y, Fu F, Zhang L. Yanshu spraying agent, a traditional Chinese medicine, relieves chronic pharyngitis in animals by anti-inflammatory and antibacterial effects. Exp Ther Med. 2014 7(4):990-4.

10. Sun Y, Zang Z, Xu X, Zhang Z, Zhong L, Zan W, Zhao Y, Sun L. Experimental investigation of the immunoregulatory and anti-inflammatory effects of the traditional Chinese medicine "Li-Yan Zhi-Ke granule" for relieving chronic pharyngitis in rats. Mol Biol Rep. 2011;38(1):199-203.

11. Qiu S-Q: Chinese medicine compound preparation for treating laryngopharyngitis and tonsillitis and its preparing method. In: Google patent. vol. CN1899501; 2007

12. Qiu S-Q, Gao H, Li Y-S: Triangular shaped lozenge of traditional Chinese medicine. In: Google patent. vol. 201230244279.X; 2012.

13. Li YS, Li WZ, Wei P, Qiu SQ. The phase II clinical trial of Yanhouqing bucca tablets in the treatment of acute pharyngitis of external wind heat type. J Clin Otolaryngol Head Neck Surg. 2016;30(15):1182-6.

14. Yi-Sheng LI, Wen-Zhou LI, Wei P, Qiu SQ. Phase III clinical trial of Yanhouqing Buccal tablets in the treatment of acute pharyngitis with external wind heat. Chin Tradit Patent Med. 2017;30(2):265-72.

15. Cheng B-H, Hu T-Y, Mo L-H, Ma L, Hu W-H, Li Y-S, Liu Z-Q, Qiu S-Q. YanHou-Qing formula attenuates allergic airway inflammation via up-regulation of Treg and suppressing Th2 responses in ovalbumin-induced asthmatic mice. J Ethnopharmacol. 2019;231:275-82.

16. Cheng B-H, Hu T-Y, Ma L, Hu W-H, Chen Y-Y, Zeng X-H, Zhao H-L, Liu Z-Q, Qiu S-Q. Anti-inflammatory action of YHQ by regulating 5-LOX/COX-2/NFKB/MAPKs/Akt signaling pathways in RAW 264.7 macrophage cells. J Herb Med. 2019;17-18(100269):1-7.

17. Sheu S-Y, Hong Y-W, Sun J-S, Liu M-H, Chen C-Y, Ke C-J. Radix Scrophulariae extracts (harpagoside) suppresses hypoxia-induced microglial activation and neurotoxicity. BMC Complement Altern Med. 2015;15(1):324.

18. Rahimi A, Razmkhah K, Mehrnia M, Mohamadnia A, Sahebjamee H, Salehi S, Asl EA, Tahmasebi H, Shandiz SAS, Davouodbeglou F, et al. Molecular docking and binding study of harpagoside and harpagide as novel antiinflammatory and anti-analgesic compound from Harpagophytum procumbens based on their interactions with COX-2 enzyme. Asian Pac J Trop Dis. 2016;6(3):227-31.

19. Abozaid OAR, Moawed FSM, Ahmed ESA, Ibrahim ZA. Cinnamic acid nanoparticles modulate redox signal and inflammatory response in gamma irradiated rats suffering from acute pancreatitis. Biochim Biophys Acta Mol basis Dis. 1866;2020(11):165904

20. Sinha S, Doble M, Manju SL. 5-Lipoxygenase as a drug target: a review on trends in inhibitors structural design, SAR and mechanism based approach. Bioorgan Med Chem. 2019;27(17):3745-59.

21. Yu T, Lao X, Zheng H. Influencing COX-2 activity by COX related pathways in inflammation and cancer. Mini-Rev Med Chem. 2016;16(15):1230-43.

22. Viswanatha GL, Thippeswamy AHM, Rafiq M, Jagadeesh M, Baig MR, Suryakanth DA, Azeemuddin M, Patki PS, Ramakrishnan S. Nove experimental model of non-infectious pharyngitis in rats. J Pharmacol Toxicolo. 2014;69(2):189-95.

23. Sun S-C. The non-canonical NF-kB pathway in immunity and inflammation. Nat Rev Immunol. 2017;17:545-58.

24. Liu T, Zhang L, Joo D, Sun S-C. NF-kB signaling in inflammation. Signal Transduct Target Ther. 2017;2(17023):1-9.
25. Arthur JSC, Ley SC. Mitogen-activated protein kinases in innate immunity. Nat Rev Immunol. 2013;13:679-92.

26. Kumar S, Boehm J, Lee JC. p38 MAP kinases: key signalling molecules as therapeutic targets for inflammatory diseases. Nat Rev Drug Discov. 2003; 2(9):717-26

27. Liu B, Bai M, Peng M, Miao M. Anti-inflammatory effect and the effect on acute pharyngitis rats model of compound Lobelia oral liquid. Saudi J Biol Sci. 2019;26(3):577-81.

28. Chen Y-H, Luo R, Lei S-S, Li B, Zhou F-C, Wang H-Y, Chen X, He X, Wang YZ, Zhan L-H, et al. Anti-inflammatory effect of Ganluyin, a Chinese classic prescription, in chronic pharyngitis rat model. BMC Complement Med Ther. 2020;20(1):265.

29. Oppong MB, Li Y, Banahene PO, Fang S-M, Qiu F. Ethnopharmacology, phytochemistry, and pharmacology of Sterculia lychnophora Hance (Pangdahai). Chin J Nat Med. 2018;16(10):721-31.

30. Shang X, Pan H, Li M, Miao X, Ding H. Lonicera japonica Thunb.: Ethnopharmacology, phytochemistry and pharmacology of an important traditional Chinese medicine. J Ethnopharmacol. 2011;138(1):1-21.

31. Chen WC, Liou SS, Tzeng TF, Lee SL, Liu IM. Wound repair and antiinflammatory potential of Lonicera japonica in excision wound-induced rats. BMC Complement Altern Med. 2012;12(226):1-9.

32. Han M-F, Zhang X, Zhang L-Q, Li Y-M. Iridoid and phenylethanol glycosides from Scrophularia umbrosa with inhibitory activity on nitric oxide production. Phytochem Lett. 2018;28:37-41.

33. Mahmood N, Nazir R, Khan M, Khaliq A, Adnan M, Ullah M, Yang H. Antibacterial activities, phytochemical screening and metal analysis of medicinal plants: traditional recipes used against diarrhea. Antibiotics. 2019; 8(194):1-16.

34. Noh D, Choi JG, Lee YB, Jang YP, Oh MS. Protective effects of Belamcandae Rhizoma against skin damage by ameliorating ultraviolet-B-induced apoptosis and collagen degradation in keratinocytes. Environ Toxicol. 2019; 34(12):1354-62.

35. Li C, Lin LM, Sui F, Wang ZM, Huo HR, Dai L, Jiang TL. Chemistry and pharmacology of Siraitia grosvenorii: a review. Chin J Nat Med. 2014;12(2): 89-102.

36. Ahn J, Kim YM, Chae HS, Choi YH, Ahn HC, Yoo H, Kang M, Kim J, Chin YW. Prenylated flavonoids from the roots and rhizomes of Sophora tonkinensis and their effects on the expression of inflammatory mediators and Proprotein Convertase Subtilisin/Kexin type 9. J Nat Prod. 2019;82(2):309-17.

37. Yu Y, Yi ZB, Liang YZ. Main antimicrobial components of Tinospora capillipes, and their mode of action against Staphylococcus aureus. FEBS Lett. 2007;581(22):4179-83.

38. Wu X, Chan SW, Ma J, Li P, Shaw PC, Lin G. Investigation of association of chemical profiles with the tracheobronchial relaxant activity of Chinese medicinal herb Beimu derived from various Fritillaria species. J Ethnopharmacol. 2018;210:39-46.

39. Ishimaru N, Maeno T, Suzuki M, Maeno T. Rapid effects of Kikyo-to on sore throat pain associated with acute upper respiratory tract infection. J Complement Integr Med. 2013;11(1):51-4.

40. Zhang L, Wang Y, Yang D, Zhang C, Zhang N, Li M, Liu Y. Platycodon grandiflorus - an ethnopharmacological, phytochemical and pharmacological review. J Ethnopharmacol. 2015;164:147-61.

41. Lan Y, Wang JY, Tao Y, Ru QG, Wang YF, Yu JX, Liu Y, Wu Q. Comparison of essential oil from Mentha haplocalyx and menthol used as penetration enhancers. Chin J Chin Mater Med. 2016:41(8):1516-22.

\section{Publisher's Note}

Springer Nature remains neutral with regard to jurisdictional claims in published maps and institutional affiliations. 